\title{
Analysis of the causes of the misdiagnosis of hereditary spherocytosis
}

\author{
SHIYUE MA ${ }^{1}$, XUELIAN DENG ${ }^{1}$, LIN LIAO $^{1}$, ZENGFU DENG $^{1}$, \\ YULING QIU ${ }^{2}$, HONGYING WEI ${ }^{2}$ and FAQUAN LIN ${ }^{1}$
}

\author{
Departments of ${ }^{1}$ Clinical Laboratory and ${ }^{2}$ Pediatric Laboratory, The First Affiliated Hospital of \\ Guangxi Medical University, Nanning, Guangxi 530021, Zhuang Autonomous Region, P.R. China
}

Received October 23, 2017; Accepted June 4, 2018

DOI: $10.3892 / o r .2018 .6578$

\begin{abstract}
Hereditary spherocy tosis (HS) is an inherited hemolytic disease with clinical diversities. The aim of the present study was to examine the reasons for prolonged misdiagnosis and mistherapy of HS in a Chinese patient, and to summarize the laboratory screening and treatment methods for this disease in increasing the knowledge towards HS. Clinical data of the proband was reviewed. The proband was first screened by detection of eosin-5'-maleimide (EMA)-labeled red blood cells (RBCs) using flow cytometry. The type of protein defect in the extracted RBC membrane proteins was confirmed by sodium dodecyl sulfate-polyacrylamide gel electrophoresis (SDS-PAGE). Mutant fragments were verified using direct DNA sequencing and matrix-assisted laser desorption/ionization time of flight (MALDI-TOF) mass spectroscopy. The proband showed a significant hemolytic tendency and significant reduction in the number of EMA-labeled RBCs. DNA sequencing indicated three site mutations in the SPTAl gene, including His54Pro, Leu1858Val and 6531-12C $>$ T. Additional DNA analysis of the three mutations in the parents of the
\end{abstract}

Correspondence to: Dr Faquan Lin, Department of Clinical Laboratory, The First Affiliated Hospital of Guangxi Medical University, 22 Shuangyong Road, Nanning, Guangxi 530021, Zhuang Autonomous Region, P.R. China

E-mail: fqlin1998@163.com

Abbreviations: EMA, eosin-5-maleimide; DBiL, direct bilirubin; HGB, haemoglobin; HS, hereditary spherocytosis; IBiL, indirect bilirubin; MALDI-TOF, matrix-assisted laser desorption/ionization time of flight; MCF, mean channel florescence; MCHC, mean corpuscular hemoglobin concentration; MCV, mean corpuscular volume; MFC, mean fluorescence channel units; MPV, mean platelet volume; MRV, mean reticulocyte volume; MSCV, mean sphered corpuscular volume; RETs, reticulocytes; RBCs, reb blood cells; RDW, red blood cell distribution width; SDS-PAGE, sodium dodecyl sulfate-polyacrylamide gel electrophoresis; TBiL, total bilirubin

Key words: hereditary spherocytosis, misdiagnosis, novel mutation, SPTAl gene, hemolytic disease proband showed that both the Leu1858Val and 6531-12C > T mutations were carried by the father and the His54Pro mutation was carried by the mother. Moreover, the mutated peptides were identified by MALDI-TOF mass spectroscopy. HS has diverse clinical manifestations and is easily missed, misdiagnosed and mistreated. Therefore, a comprehensive analysis involving a routine blood test, blood smear, EMA labeling (flow cytometry) and SDS-PAGE can effectively distinguish HS from thalassemia, glucose-6-phosphate deficiency, iron-deficiency anemia and autoimmune hemolytic anemia.

\section{Introduction}

Hereditary spherocytosis (HS) is a type of hemolytic anemia caused by abnormal red blood cell (RBC) membrane proteins, and approximately $75 \%$ patients are autosomal dominant for the disease (1). HS is found globally at a different prevalence in different countries. The prevalence of HS is as high as 1/2000 in northern Europe and North America (2). Although anemia, jaundice and splenomegaly are typical clinical manifestations of HS, due to the different types of membrane protein defects, clinically the disease is still highly heterogeneous. In approximatelly $20-30 \%$ of HS patients, clinical manifestations are not obvious, except for a compensatory increase in reticulocytes (RETs) (3). In these patients, hemolytic symptoms are aggravated when certain factors are induced, the most common of which are infections or continuous heavy physical activities. Moreover, severe HS patients often present with severe hemolysis, and require blood transfusions to maintain a hemoglobin (HGB) content of $>60 \mathrm{~g} / \mathrm{l}$. However, this procedure can often lead to iron overload or other complications in these patients. Although HS is not an uncommon clinical disease, its definitive diagnosis rate remains relatively low. The lack of knowledge among Chinese physicians towards the disease is one of the main reasons of misdiagnosis and mistherapy of many HS patients. This study reports a case of severe HS that has been misdiagnosed for up to 9 years. The aim of the present study was to investigate the factors that led to the long-term misdiagnosis of the disease, and to provide the proband with reasonable treatment recommendations based on the international principles of HS treatment. 


\section{Materials and methods}

Routine examination. Peripheral venous blood $(2 \mathrm{ml})$ was collected from the proband and the proband's family into tubes containing the anticoagulant EDTA- $\mathrm{K}_{2}$. Routine blood tests, including RBC, HGB, mean corpuscular volume (MCV), mean corpuscular hemoglobin $(\mathrm{MCH})$, mean sphered cell volume (MSCV), mean reticulocyte volume (MRV), RET and mean platelet volume (MPV), were performed using the LH 780 automated hematology analyzer (Beckman Coulter, Carlsbad, CA, USA). Liver function, especially total bilirubin (TBiL) and direct bilirubin (DBiL), was assessed using the 7600 fully automated biochemical analyzer (Hitachi, Ltd., Tokyo, Japan). This study procured the informed consents of the proband and his family members, and the approval of the Ethics Committee of The First Affiliated Hospital of Guangxi Medical University (Nanning, China). From all participants, written informed consent for molecular genetic analysis, data analysis and publication was obtained.

Flow cytometric analysis of EMA-labeled red blood cells. EMA working solution, at a final concentration of $0.5 \mathrm{mg} / \mathrm{ml}$, was prepared by diluting EMA powder in phosphate-buffered saline (PBS; Sigma-Aldrich Co., Dorset, UK). The EMA solution was stored at $-20^{\circ} \mathrm{C}$ in the dark and thawed at $4^{\circ} \mathrm{C}$ at the time of use. PBS-washed RBCs $(5 \mu \mathrm{l})$ were incubated with $20 \mu 1$ EMA working solution for $1 \mathrm{~h}$ at room temperature in the dark, and then washed 3 times with PBS containing 0.5\% fetal bovine serum (FBS; Life Technologies Corp., Shanghai, China). The mixture was centrifuged for $5 \mathrm{~min}$ at $2,500 \mathrm{x} \mathrm{g}$ to remove the supernatant, and the pellet was resuspended in $600 \mu$ l PBS for flow cytometric analysis (Beckman Coulter, High Wycombe, UK) (4). The flow cytometer was calibrated by recording the fluorescence intensity of 10,000 cells in the FL-1 channel prior to use. Data were analyzed using Flowjo software (FlowJo, LLC, Ashland, OR, USA).

Sodium dodecyl sulfate-polyacrylamide gel electrophoresis. RBC membrane proteins were extracted as per Rungaldier's methods (5), and 3.5-17\% sodium dodecyl sulfate-polyacrylamide gel electrophoresis (SDS-PAGE) was prepared based on the protocol of Fairbanks et al (6). An RBC membrane protein precipitate was mixed with $\mathrm{RBC}$ membrane lysis solution, containing mercaptoethanol, and incubated for $1 \mathrm{~h}$ in a $37^{\circ} \mathrm{C}$ waterbath. The proteins were then separated via SDS-PAGE, along with a broad range protein ladder (Bio-Rad Laboratories, Hercules, CA, USA) to indicate the molecular size of the unknown proteins. Upon Coomassie blue staining, protein bands in the gel were scanned using a Gel Doc 2000 Imaging System (Bio-Rad Laboratories), and protein defects were analyzed using Quantity One quantification analysis software (Bio-Rad Laboratories).

Mass spectrometry. In-gel digestion was performed as per the methods described by Gharahdaghi et al (7). Briefly, the protein samples from the proband and healthy controls were separated by SDS-PAGE and stained with Coomassie blue. Target proteins were then removed from the gel and placed into 1.5-ml microeppendorf tubes. The protein-containing gel strips were washed twice by water and decolorized, and dehydrated

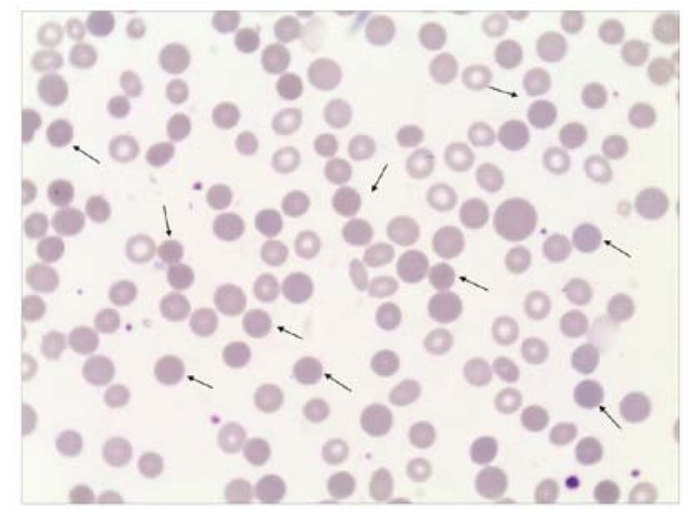

Figure 1. Morphology of peripheral blood spherocytes of the proband. Arrows indicate spherocytes.

Table I. Laboratory test results of the HS proband and his family members.

\begin{tabular}{lcccc}
\hline Item & Proband & Father & Mother & Normal range \\
\hline RBC $\left(\times 10^{12} / \mathrm{l}\right)$ & 1.69 & 4.93 & 4.8 & $3.5-5.5$ \\
$\mathrm{Hb}(\mathrm{g} / \mathrm{l})$ & 48.9 & 149.2 & 134.4 & $110.0-160.0$ \\
$\mathrm{MCV}(\mathrm{fl})$ & 79.69 & 93.77 & 85.81 & $82.00-95.00$ \\
$\mathrm{MCHC}(\mathrm{g} / \mathrm{l})$ & 360.8 & 322.6 & 326.1 & $316.0-354.0$ \\
$\mathrm{MSCV}(\mathrm{fl})$ & 75.68 & 96.38 & 80.59 & $84.00-104.00$ \\
$\mathrm{MRV}(\mathrm{fl})$ & 89.30 & 112.18 & 106.68 & $101.00-119.00$ \\
Ret $(\%)$ & 7 & 1.4 & 0.9 & $0.0-2.0$ \\
TBIL $(\mu \mathrm{mol} / \mathrm{l})$ & 178.10 & 15.6 & 10.4 & $3.40-20.50$ \\
DBIL $(\mu \mathrm{mol} / \mathrm{l})$ & 59.20 & 5.5 & 3.0 & $0.00-6.8$ \\
\hline
\end{tabular}

RBC, red blood cell count; Hb, hemoglobin; MCV, mean corpuscular volume; MCHC, mean corpuscular hemoglobin concentration; MSCV, mean sphered corpuscular volume; MRV, mean reticulocyte volume; Ret \%, reticulocyte ratio; TBIL, total bilirubin; DBIL, direct bilirubin.

in pure acetonitrile (ACN) and dried under vacuum. Gel strips were digested overnight at room temperature using trypsin and $30 \mu \mathrm{l}$ digestion buffer $\left(40 \mathrm{mM} \mathrm{NH}_{4} \mathrm{HCO}_{3}\right.$ and $\left.10 \% \mathrm{ACN}\right)$, and extracted peptides were centrifuged for $5 \mathrm{~min}$ at $10,000 \mathrm{x} \mathrm{g}$. The upper layer of the solution was concentrated by vacuum drying, and the precipitate was resuspended in $0.1 \%$ trifluoroacetic acid solution. The extracted proteins were analyzed using the 4800 Plus System Mass spectrometer (AB SCIEX, Boston, MA, USA).

Gene sequence analysis. Peripheral blood DNA of the proband and his family members was extracted using a DNA extraction kit (Tiangen Biotech Co., Ltd., Beijing, China). All relevant primers for the exons, introns and flanking sequences of the gene were designed by the Primer Premier 5.0 software, and synthesized by Beijing Genomics Institute (BGI; Beijing, China). The sequences of target mutations were as follows: exon 2, forward 5'-ACACATATAAGCGGGGCAAC-3', reverse 5'-TTGTACCCACACATACCCATTAAC-3'; exon 40, forward 5'-TGAGTGAATATAGATTTTCCGGC-3', reverse 
$\mathbf{A}$

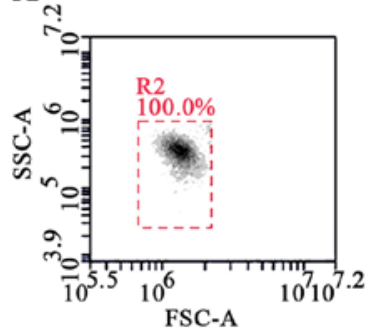

B-1

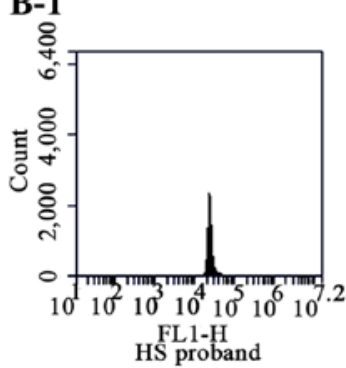

B-2

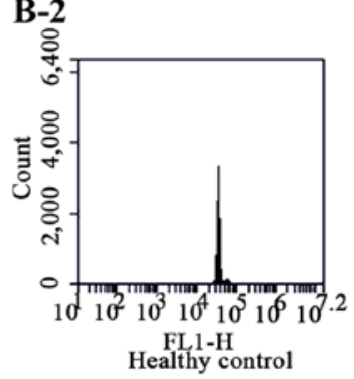

C

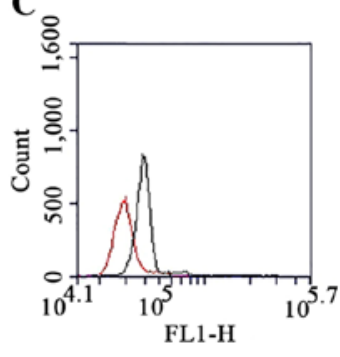

Figure 2. Flow cytometric evaluation of eosin-5'-maleimide (EMA)-labeled red blood cells. (A) Basic side scatter/forward scatter cytograms of red blood cells (RBCs) stained with EMA. (B) The fluorescence of EMA-stained RBCs from the (B-1) proband and (B-2) healthy controls. (C) Overlay of plots comparing the fluorescence of RBCs from the patient with that of the healthy controls.

5'-TTCTACATTTGGGCCAGTCC-3'; intron 45, forward 5'-TGGACAGATTCATGTTTTGTGG-3', reverse 5'-TGGAA TGAAAATGTCTCAGCAC-3'. PCR amplification was performed using an Applied Biosystems 9700 Thermal Cycler (Thermo Fisher Scientific, Inc., Waltham, MA, USA) in a 50- $\mu$ l reaction, containing $25 \mu$ l PCR Master Mix (Takara Bio, Otsu, Japan), $1 \mu \mathrm{l}$ each of forward and reverse primers, $0.2 \mathrm{ng}$ DNA and double-distilled water. Amplification was conducted at $95^{\circ} \mathrm{C}$ for $5 \mathrm{~min}, 95^{\circ} \mathrm{C}$ for $30 \mathrm{sec}$, annealing for $30 \mathrm{sec}$, and $72^{\circ} \mathrm{C}$ for $40 \mathrm{sec}$ for a total of 35 cycles, followed by $72^{\circ} \mathrm{C}$ for $8 \mathrm{~min}$ and $4^{\circ} \mathrm{C}$ hold. PCR products were recovered, purified from the gel using the Agarose DNA Purification kit (Solarbio Life Science, Shanghai, China) and sequenced by the ABI3730XL sequencer (Thermo Fisher Scientific, Inc.). All variations were classified as pathogenic, potentially pathogenic, undefined, potentially benign and benign according to the American College of Medical Genetics and Genomics (ACMG) guidelines and recommendations (8-10).

\section{Results}

Patient and routine blood tests. The proband (male, born in 2006, Han nationality, Guangxi residence, China) presented with jaundice on the day of birth, and had $401.5 \mu \mathrm{mol} / \mathrm{l}$ of TBiL. The proband was diagnosed with neonatal hyperbilirubinemia, but did not undergo exchange transfusion. At 4 months of age, the proband appeared pale and dispirited with no obvious presence of inducers. He was diagnosed with severe anaemia, and received repeated transfusion therapy at a local hospital at an average of one transfusion per month. At 2 years of age, the proband was admitted to the hospital because of a fever (up to $38.8^{\circ} \mathrm{C}$ ) that lasted 3 days and was induced by bronchopneumonia. Moreover, the proband showed symptoms resembling thalassemia and was diagnosed with thalassemia and G6PD deficiency. Laboratory test results indicated 2.64x 10 $12 / 1 \mathrm{RBC}, 54 \mathrm{~g} / 1$ haemoglobin (HGB), $68.00 \mathrm{fl}$ MCV, $277.0 \mathrm{~g} / 1$ mean corpuscular hemoglobin concentration (MCHC), $47.3 \mu \mathrm{mol} / 1 \mathrm{TBiL}$ and $15.9 \mu \mathrm{mol} / 1 \mathrm{DBiL}$. The proband was given antitussive and antipyretic medication but did not show any improvement. Upon antiviral and anti-infection therapy, the body temperature of the proband returned to normal and coughing disappeared. Routine blood tests revealed mild anemia, suggesting improvement of the thalassemia-like illness. At 8 years of age, the proband was admitted to a Hospital located in Nanning due to acute upper respiratory tract infections accompanied by breathing difficulties. Laboratory test results indicated $1.04 \times 10^{12} / 1 \mathrm{RBC}, 30 \mathrm{~g} / \mathrm{l}$ HGB, $316.70 \mu \mathrm{mol} / 1 \mathrm{TBiL}, 109.52 \mu \mathrm{mol} / 1 \mathrm{DBiL}, 207.2 \mu \mathrm{mol} / 1$ IBiL, 3+ urinary bilirubin (BiL) and 3+ urinary bile, therefore, the proband was diagnosed with chronic hemolytic anemia and anemic cardiomyopathy. In 2015, the proband came to our hospital for examination due to ' 9 years of jaundice and 2 days of fever', without any history of genetic disorders. Physical examination showed that the proband was clearly conscious but low-spirited, and had jaundice in the sclera and on the skin, along with hepatosplenomegaly. Laboratory test results demonstrated that the proband was negative for the Coombs test, autoantibody, and hepatitis B surface antigen, and had a high level of serum ferritin $(3503 \mathrm{ng} / \mathrm{ml})$, normal immunoglobulin levels, normal genetic testing for thalassemia, normal G6PD activity, and increased erythrocyte osmotic fragility and spherocytes (25\% of all RBCs) (Fig. 1). In consideration of his medical history, clinical manifestations and laboratory test results, the proband was finally diagnosed with HS. Upon completion of relevant examinations and preoperative preparations, the proband underwent splenectomy under general anesthesia, and was given anti-inflammatory, hemostatic and transfusion therapies after the operation. The proband was discharged from the hospital once his vital signs had stabilized. Routine blood and biochemical tests were conducted on the proband's family members and the results are presented in Table I.

Fluorescence results for red blood cells from the patient and healthy controls. Blood was collected from 10 healthy individuals that were age and sex matched with the proband, and was used as negative controls to reduce the errors between samples (11). A blank control was also included. Fluorescence of EMA-labeled RBCs was expressed as mean fluorescence channel units (MFC). The percent reduction in MCF of the proband was calculated as follows:

$\mathrm{MCF}(\%)=\frac{M C F \text { of healthy controls }-M C F \text { of proband }}{M C F \text { healthy controls }} \times 100 \%$

It was found that the MCF of the proband's blood was reduced by $35.77 \%$ compared with those of the healthy controls (Fig. 2).

Analysis of red blood cell membrane proteins. RBC membrane proteins of the proband were evaluated by SDS-PAGE and Commassie brilliant blue staining. The proband and his family 


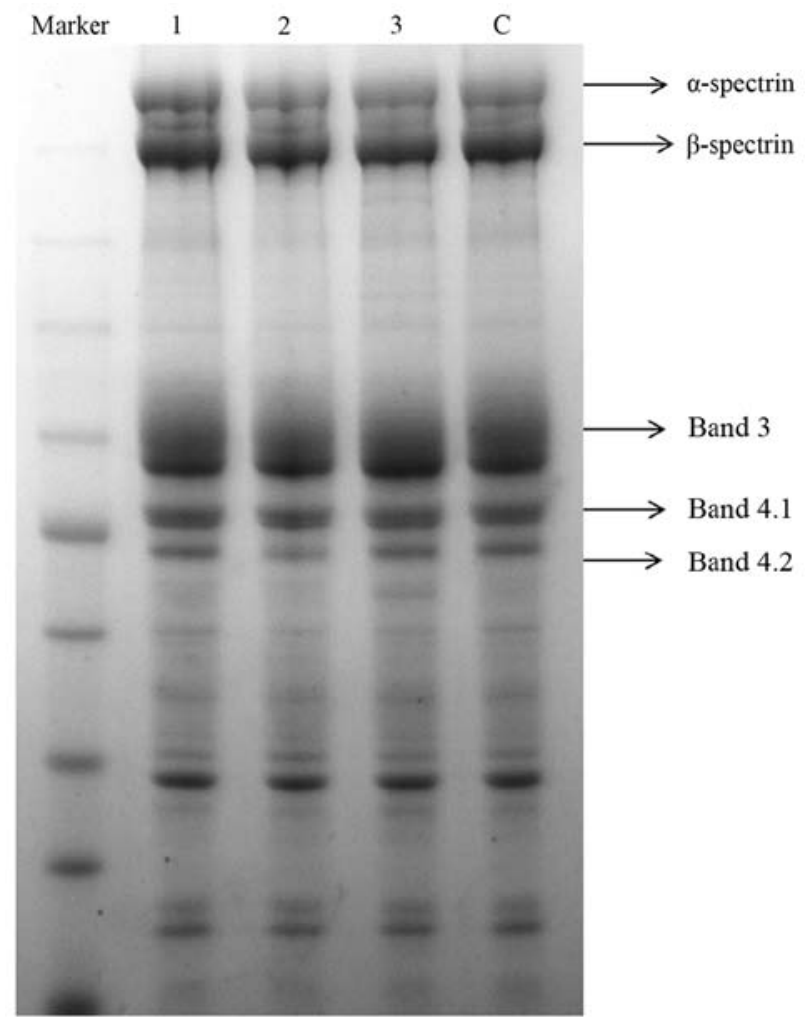

Figure 3. Proteins were separated by SDS-PAGE using 5\% (w/v) stacking gels and $3.5-17 \%(\mathrm{w} / \mathrm{v})$ polyacrylamide gels under reducing conditions. Lanes $1,2,3$ and $\mathrm{C}$ correspond to red blood cell membrane protein precipitation from plasma of the patient, the patient's father, the patient's mother and a healthy control individual, respectively.

did not show any significant reduction in membrane protein levels compared with that of the healthy controls (Fig. 3).

Matrix-assisted laser desorption/ionization time of flight mass spectrometry (MALDI-TOF MS). The $\alpha$-spectrin peptide chain of the proband was analyzed using MALDI-TOF MS. When comparing the $\alpha$-spectrin peptide chain of the proband with that of reference peptides in the database it was found that the molecular sizes of RBC $\alpha$-spectrin in the proband were 1,338.52 Da (LEDSYHLQVFK) and $1,609.83 \mathrm{Da}$ (GDCGDTLAATQSLVMK) as opposed to $1,378.55 \mathrm{Da}$ (LEDSYPLQVFK) and 1,623.86 $\mathrm{Da}$ (GDCGDTLAATQSLLMK) of normal $\alpha$-spectrin. This indicated that the $\alpha$-spectrin chain of the proband contained two abnormal fragments (Fig. 4).

DNA analysis. Human RBC membrane genes SPTA1, SPTB, SLC4A1, ANKI and EPB42 were amplified by PCR and analyzed by genomic DNA sequencing (Fig. 5). Comparing the sequencing results to the reference sequences in the NCBI database showed that the proband carried 3 mutations, including c.161 $\mathrm{A}>\mathrm{C}$ heterozygous missense mutation in exon 2 of the SPTAl gene, leading to the substitution of histidine (His) by proline (Pro) at position 54 of the $\alpha$-spectrin peptide; c.5572C $>\mathrm{G}$ heterozygous missense mutation in exon 40 of the SPTA gene, leading to the substitution of leucine (Leu) by valine (Val) at position 1858; and a $6531-12 \mathrm{C}>\mathrm{T}$ heterozygous mutation in intron 45. Mutation analysis of the proband's family members showed that the His54Pro mutation was present in the mother, whereas both Leu1858Val and 6531-12C $>\mathrm{T}$ mutations were present in the father (Fig. 6).

\section{Discussion}

Hereditary spherocytosis (HS) has very diverse clinical manifestations, which typically include anemia, jaundice and splenomegaly. Common complications include cholecystolithiasis, hemolytic crisis, hematopoietic crisis and are sometimes combined with delayed growth, heart diseases and neuromuscular disorders. Laboratory test results of HS generally show increases in reticulocytes (RETs) with reduced volume, peripheral spherocytes, RBC osmotic fragility, mean corpuscular hemoglobin concentration (MCHC) and red blood cell distribution width (RDW) (3). Therefore, timely and accurate diagnosis of HS is critical for optimal treatment regimen and the prognosis of the patient. The Guidelines for the Diagnosis and Management of HS that was developed by the British Committee for Standards in Hematology stated that an individual can be definitively diagnosed with HS without further testing if he/she has a family history of HS, and presents with typical clinical and blood characteristics of HS (12). However, a negative family history was one of the main reasons for misdiagnosis of the proband. It has been shown that many mild HS patients have no obvious clinical symptoms, which makes it especially difficult to diagnose the disease using conventional methods (13). This was particularly true in case of the proband's mother, as the size and morphology of mature RBCs in her peripheral blood were relatively consistent with almost no signs of abnormality, and HS could only be identified through a genetic testing approach. Another possibility would be that HS was recessively inherited through the family, and individuals with recessive inheritance or serendipitous mutation have no family history of the disease. Thus, these possibilities may all have resulted in the prolonged misdiagnosis and mistherapy of the patient.

Identification of a suitable laboratory test has important implication in the improvement of HS diagnosis. Since anemia, jaundice and splenomegaly are all clinical manifestations of G6PD deficiency, thalassemia, and AIHA, and given that thalassemia is highly prevalent in regions of the Guangxi Province, it is easy to confuse HS with these diseases. When a patient is suspected of having hemolytic anemia, examination of peripheral RBC morphology, RBC protein electrophoresis, genetic analysis, detection of G6PD levels, and a Coombs test can all help distinguish HS from these diseases (14). In particular, the concurrent use of genetic analysis and RBC protein electrophoresis is a conventional means for the diagnosis of thalassemia in Guangxi regions. In addition, routine blood testing is also a common conventional measure for HS screening. In a study by Broséus et al (15) it was reported that MCV $<80 \mathrm{f} 1$, MCHC $>354 \mathrm{~g} / \mathrm{l}$ and RDW $>14 \%$ in routine peripheral blood tests showed a $63 \%$ sensitivity and a $100 \%$ specificity for the diagnosis of HS. Subsequently, Liao et al (16) found that both sensitivity and specificity of MCHC were undesirable for the diagnosis of HS in patients with increased RET levels, and that increased blood TBiL levels could interfere with the detection of HGB and result in a false increase in MCHC (17). On the other hand, MSCV is a valuable indicator for HS 

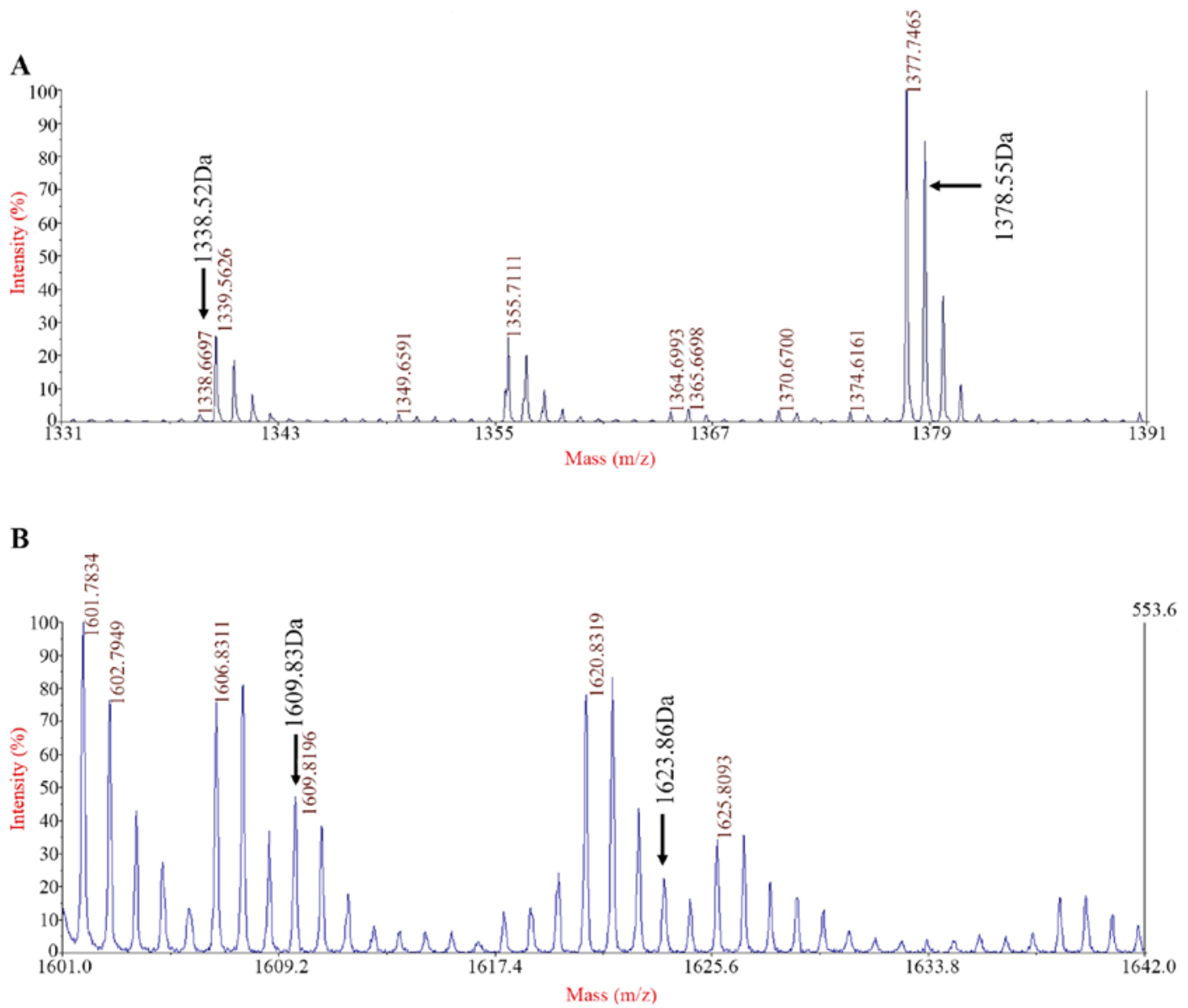

Figure 4. Mass spectrometry analysis of the proband's red blood cell membrane protein fragments. Peaks indicate the normal peptides (1,378.55 Da, LEDSYPLQVFK and 1,623.86 Da, GDCGDTLAATQSLLMK) and variant peptides (1,338.52 Da, LEDSYHLQVFK and 1,609.83 Da, GDCGDTLAATQSLVMK).

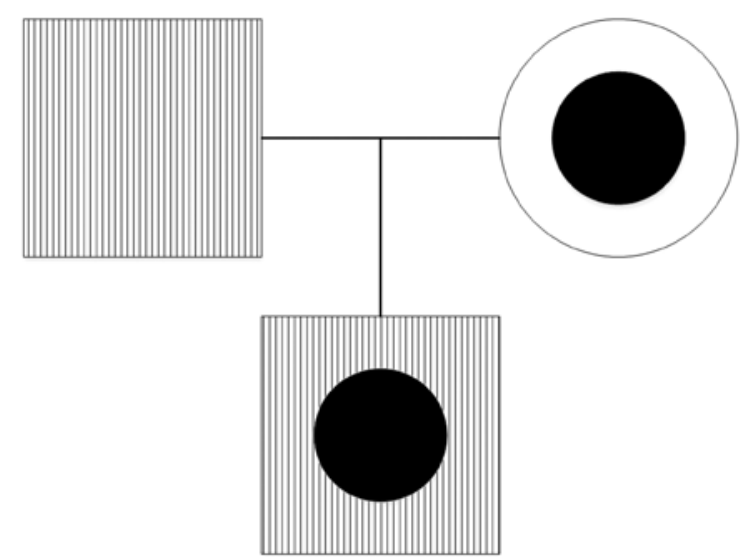

Figure 5. Pedigree of the hereditary spherocytosis (HS) family under study. The square with vertical lines denotes members with c.5572C $>\mathrm{G}$ and $6531-12$ $\mathrm{C}>\mathrm{T}$ mutations. The black circle in the white circle denotes members with c.161A $>$ C mutation in exon 2 .

screening (15). A study in $57 \mathrm{HS}$ patients and 109 thalassemia patients by Liao et al (16) showed that MSCV<MCV could effectively distinguish HS from thalassemia. Furthermore, Tao et al (18) reported that the combination of $\mathrm{MSCV}<\mathrm{MCV}$, flow cytometry and osmotic fragility test demonstrated $89.28 \%$ sensitivity and $96.14 \%$ specificity for the diagnosis of H. MRV is a novel indicator for HS screening. The value of MRV in the screening of HS was first reported by Lazarova et al (19) in 2014, where they demonstrated that MRV $\leq 96.72 \mathrm{fl}$ showed $100 \%$ sensitivity and $88 \%$ specificity for the diagnosis of HS. Moreover, in a study performed on 53 HS patients, 109 thalassemia patients, 56 G6PD deficiency patients, and 52 AIHA patients by $\mathrm{Xu}$ et al (20) it was demonstrated that MRV $\leq 95.77$ fl showed $86.8 \%$ sensitivity and $91.2 \%$ specificity for the diagnosis of HS. The limitations of basic hospitals and insufficient knowledge of clinicians towards HS were the main reasons for misdiagnosis of the proband. The proband was first diagnosed with thalassemia and G6PD deficiency by a local hospital. At the first time of diagnosis, HGB electrophoresis and G6PD detection were not conducted. Thus, the proband was diagnosed with hemolytic anemia at another hospital in Nanning, China. It is important to note that at the time of his second diagnosis, the RBC parameters of the proband were typical, where pre-treatment values of MCV, MCHC, MRV and MSCV all met the diagnostic criteria for HS. Therefore, diagnosis of the patient would not have been difficult if the clinician had performed reasonable comprehensive analyses and further diagnostic testing.

The undesirable sensitivity and specificity of traditional HS screening methods are also a reason of prolonged misdiagnosis or missed diagnosis of several HS patients (21). SDS-PAGE is mainly used for the detection of changes in 


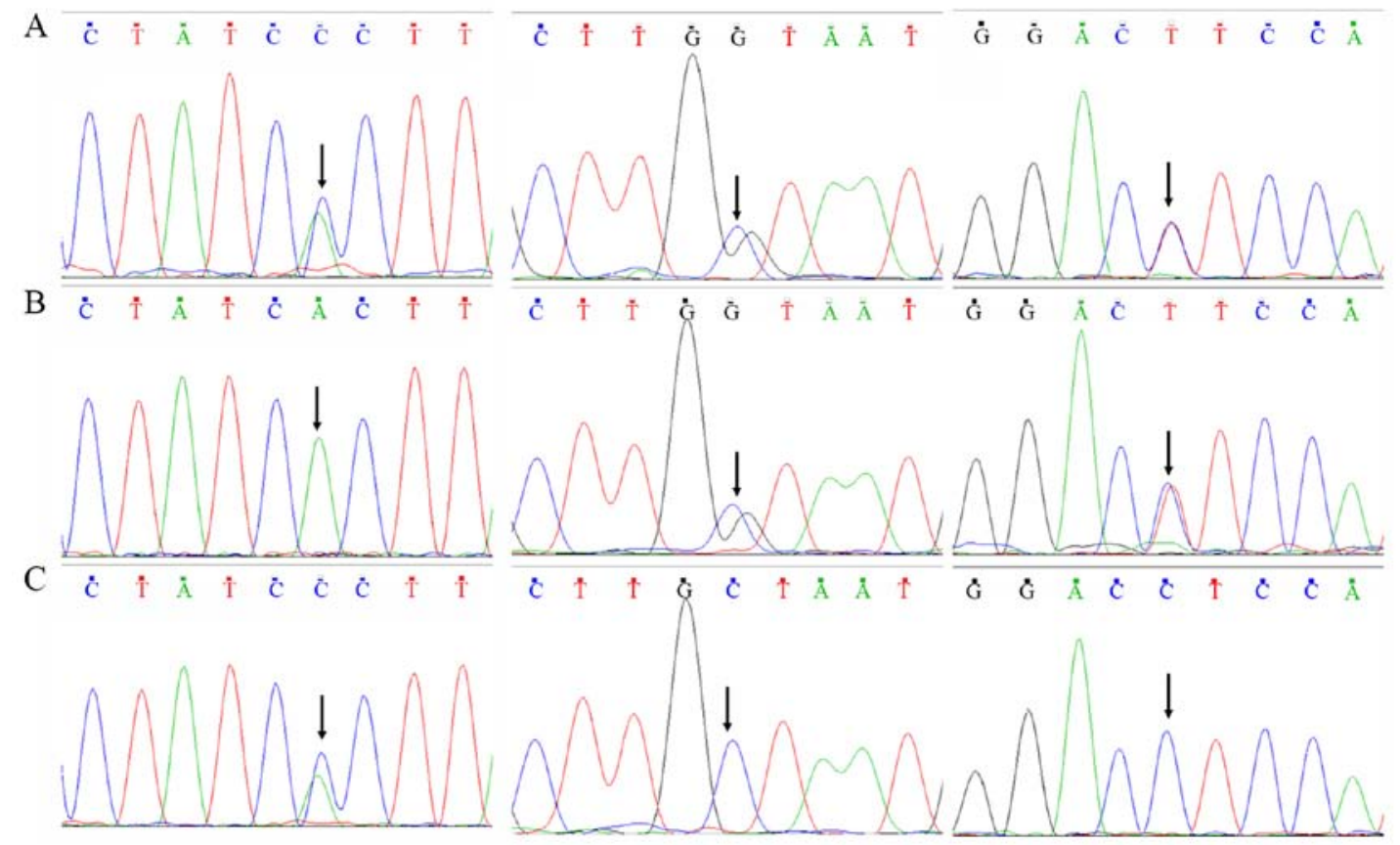

Figure 6. DNA sequences indicate from top to bottom: proband, father, and mother. (A) Heterozygous point mutation $\mathrm{A} \rightarrow \mathrm{C}$ was found in exon2 of the proband and his mother. (B) Heterozygous point mutation $C \rightarrow G$ was found in exon 40 of the proband and his father. (C) Heterozygous point mutation $C \rightarrow T$ was found in intron 45 of the proband and his father.

the relative levels of $\mathrm{RBC}$ membrane proteins in $\mathrm{HS}$ patients compared to healthy controls. The SDS-PAGE approach can be used to detect the presence of abnormal proteins in roughly $80 \%$ of HS patients, however, the type of protein defect cannot be confirmed in approximately $10 \%$ of HS patients (22). In this study, membrane protein defects in the proband were not detected by SDS-PAGE because the excess amount of ankyrin proteins in the RETs interfered with the gradient of the gel and the separation of RBC membrane proteins.

Detection of EMA-labeled RBCs by flow cytometry is a novel method of preliminary screening for HS, especially in atypical patients (23). EMA is a fluorescent probe that covalently binds to Lys-430 on the first extracellular ring of band 3 protein and spectrin (24). Upon EMA labeling, the MCF of RBCs is reduced, and $>21 \%$ reduction in MCF was found to show $97 \%$ sensitivity and $100 \%$ specificity for the diagnosis of HS (25). This approach is fast, requires a small amount of blood, and is therefore, a superior auxiliary method for the clinical diagnosis of HS.

HS is caused by the deficiency or functional change of $\mathrm{RBC}$ membrane proteins resulting from genetic mutations. As the shape-changing ability of RBCs is reduced, and upon entering the microcirculation of the spleen, these cells are easily captured for phagocytosis. The main RBC membrane protein-encoding genes include SPTA1, SPTB, ANK1, $S L C 4 A 1$ and $E P B 42$, and the corresponding proteins they encode include $\alpha$-spectrin, $\beta$-spectrin, ankyrin, band 3 and 4.2 protein, respectively. Previous studies have shown that the severity of HS is associated with the type of membrane protein defect in the patient (26). Specifically, patients with defects in $\alpha$-spectrin present with severe anemia, while those with defects in ankyrin, band 3 and 4.2 protein present with mild to moderate anemia (27).
It is found that approximately $5 \%$ of HS patients have a defect in $\alpha$-spectrin and they acquired the disease by recessive inheritance (28). Generally, a single heterozygous mutation in the SPTAl gene will not lead to protein defects or the appearance of obvious clinical symptoms in the patients, because overexpression of $\alpha$-spectrin will maintain protein levels. Only homozygous or compound heterozygous mutations will result in disease (29). The proband was identified as a carrier for compound heterozygous mutations in the SPTAl gene. In addition, 3 site mutations were found in the proband, including c. $161 \mathrm{~A}>\mathrm{C}$ in exon $2, \mathrm{c} .5572 \mathrm{C}>\mathrm{G}$ in exon 40 , and $6531-12 \mathrm{C}>\mathrm{T}$ in intron 45 . In particular, c.161 $\mathrm{A}>\mathrm{C}$ was a newly identified mutation that had not yet been reported in the dbSNP database, and hence there is no information available regarding the mutation in the 1000 Genomes dataset, such as its distribution frequency and the mechanism of pathogenesis. Harmfulness prediction was made through the web PolyPhen-2 (genetics.bwh.harvard. edu/pph2/) (30) and Mutation Taster (www.mutationtaster. org/). The results showed that $\mathrm{c} .161 \mathrm{~A}>\mathrm{C}$ is pathogenic or potentially pathogenic according to the ACMG guidelines and recommendations. We speculated that the mutation may affect the level of mRNA expression or the structural stability of proteins. In addition, c.5572C $>\mathrm{G}$ and $6531-12 \mathrm{C}>\mathrm{T}$ together result in a polymorphic 'LELY' allele that is highly frequent but lowly expressed (31). The 6531-12C $>\mathrm{T}$ mutation can cause $50 \%$ of the transcription codons to be skipped during translation of exon 46, resulting in the deficiency of 6 amino acids that link $\alpha$-spectrin with $\beta$-spectrin. The c.5572C $>\mathrm{G}$ mutation can lead to amino acid changes and linkage disequilibrium with 6531-12C >T. In general, a single heterozygous mutation (mother)

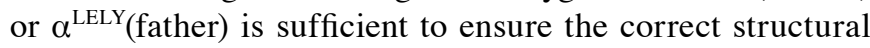
expression of the $\alpha$-spectrin protein and prevents the development of corresponding pathological changes in the carrier. 
Even if an individual develops relevant pathological changes, the damage is very minimal (32). Simultaneous inheritance of both alleles in the proband resulted in significant anemia due to the vicious interaction between the two mutations, in which the presence of $\alpha^{\mathrm{LELY}}$ exacerbated the pathogenesis of c.161A $>C$, and the presence of c.161A $>C$ exacerbated the trans effect of $\alpha^{\text {LELY }}$ on the structure of $\alpha$-spectrin (33).

In conclusion, although HS is not an uncommon or rare disorder, its sporadic nature and the absence of reliable laboratory indicators for definitive diagnosis of HS prompt the development of a comprehensive assessment involving multiple laboratory indicators that will allow for accurate diagnosis of the disease. EMA-labeled RBC detection by flow cytometry, SDS-PAGE, and an osmotic fragility test can be used concurrently to determine the fragility, and the presence of membrane protein defects in RBCs. Furthermore, performance of a blood smear and routine laboratory tests, such as RBC, HBG, MCV, MSCV, RET\%, MRV, TBiL and DBiL, can effectively distinguish HS from thalassemia, G6PD deficiency, iron-deficiency anemia and AIHA.

\section{Acknowledgements}

Not applicable.

\section{Funding}

The present study was supported by the National Natural Science Foundation of China (no. 81360263).

\section{Availability of data and materials}

The datasets supporting the conclusions of this article are included within the article.

\section{Authors' contributions}

SM drafted the initial manuscript and organized the data and contributed to the design of the report and to the critical writing of the manuscript. HW is a physician involved in the care of the patient in the Pediatric Hematology Clinic. YQ is the principal physician caring for the hematological issues of this patient. XD, LL and ZD, research scientists at Clinical Laboratory, conceived of and established the flow-cytometric studies and the gene sequencing aspects that are central to this report. FL, a senior hematologist and scientist, has been involved as a consultant on the clinical case and supervises the scientists in aspects of hematology and genetics. He provided critical advice in case management, contributed to the design and critically reviewed the manuscript. All authors read and approved the manuscript and agree to be accountable for all aspects of the research in ensuring that the accuracy or integrity of any part of the work are appropriately investigated and resolved.

\section{Ethics approval and consent to participate}

Written informed consent was obtained from all participants in this study. The study protocol was approved by the Ethics Committee of the First Affiliated Hospital at the Guangxi Medical University in China.

\section{Patient consent for publication}

This study procured the informed consents of the proband and his family members. From all participants, written informed consent for molecular genetic analysis, data analysis and publication was obtained.

\section{Competing interests}

The authors declare that they have no competing interests.

\section{References}

1. Yawata Y, Kanzaki A, Yawata A, Doerfler W, Ozcan R and Eber SW: Characteristic features of the genotype and phenotype of hereditary spherocytosis in the Japanese population. Int $\mathbf{J}$ Hematol 71: 118-135, 2000.

2. Bolton-Maggs PH, Langer JC, Iolascon A, Tittensor P and King MJ; General Haematology Task Force of the British Committee for Standards in Haematology: Guidelines for the diagnosis and management of hereditary spherocytosis - 2011 update. Br J Haematol 156: 37-49, 2012

3. King MJ, Garçon L, Hoyer JD, Iolascon A, Picard V, Stewart G, Bianchi P, Lee SH and Zanella A; International Council for Standardization in Haematology: ICSH guidelines for the laboratory diagnosis of nonimmune hereditary red cell membrane disorders. Int J Lab Hematol 37: 304-325, 2015.

4. Ciepiela O, Kotuła I, Górska E, Stelmaszczyk-Emmel A, Popko K, Szmydki-Baran A, Adamowicz-Salach A and Demkow U: Delay in the measurement of eosin-5'-maleimide (EMA) binding does not affect the test result for the diagnosis of hereditary spherocytosis. Clin Chem Lab Med 51: 817-823, 2013.

5. Rungaldier S, Oberwagner W, Salzer U, Csaszar E and Prohaska R: Stomatin interacts with GLUT1/SLC2A1, band 3/ SLC4A1, and aquaporin-1 in human erythrocyte membrane domains. Biochim Biophys Acta 1828: 956-966, 2013.

6. Fairbanks G, Steck TL and Wallach DF: Electrophoretic analysis of the major polypeptides of the human erythrocyte membrane. Biochemistry 10: 2606-2617, 1971.

7. Gharahdaghi F, Weinberg CR, Meagher DA, Imai BS and Mische SM: Mass spectrometric identification of proteins from silver-stained polyacrylamide gel: A method for the removal of silver ions to enhance sensitivity. Electrophoresis 20: 601-605, 1999.

8. Richards CS, Bale S, Bellissimo DB, Das S, Grody WW, Hegde MR, Lyon E and Ward BE; Molecular Subcommittee of the ACMG Laboratory Quality Assurance Committee: ACMG recommendations for standards for interpretation and reporting of sequence variations: Revisions 2007. Genet Med 10: 294-300, 2008.

9. Richards S, Aziz N, Bale S, Bick D, Das S, Gastier-Foster J, Grody WW, Hegde M, Lyon E, Spector E, et al; ACMG Laboratory Quality Assurance Committee: Standards and guidelines for the interpretation of sequence variants: a joint consensus recommendation of the American College of Medical Genetics and Genomics and the Association for Molecular Pathology. Genet Med 17: 405-424, 2015.

10. Li MM, Datto M, Duncavage EJ, Kulkarni S, Lindeman NI, Roy S, Tsimberidou AM, Vnencak-Jones CL, Wolff DJ, Younes A, et al: Standards and guidelines for the interpretation and reporting of sequence variants in cancer: A joint consensus recommendation of the Association for Molecular Pathology, American Society of Clinical Oncology, and College of American Pathologists. J Mol Diagn 19: 4-23, 2017.

11. Hunt L, Greenwood D, Heimpel H, Noel N, Whiteway A and King MJ: Toward the harmonization of result presentation for the eosin-5'-maleimide binding test in the diagnosis of hereditary spherocytosis. Cytometry B Clin Cytom 88: 50-57, 2015.

12. Manciu S, Matei E and Trandafir B: Hereditary spherocytosis diagnosis, surgical treatment and outcomes. A literature review. Chirurgia (Bucur) 112: 110-116, 2017.

13. Barcellini W, Bianchi P, Fermo E, Imperiali FG, Marcello AP, Vercellati C, Zaninoni A and Zanella A: Hereditary red cell membrane defects: Diagnostic and clinical aspects. Blood Transfus 9: 274-277, 2011. 
14. Guillaud C, Loustau V and Michel M: Hemolytic anemia in adults: Main causes and diagnostic procedures. Expert Rev Hematol 5: 229-241, 2012.

15. Broséus J, Visomblain B, Guy J, Maynadié M and Girodon F: Evaluation of mean sphered corpuscular volume for predicting hereditary spherocytosis. Int J Lab Hematol 32: 519-523, 2010.

16. Liao L, Deng ZF, Qiu YL, Chen P, Chen WQ and Lin FQ: Values of mean cell volume and mean sphered cell volume can differentiate hereditary spherocytosis and thalassemia. Hematology 19: 393-396, 2014.

17. Kar R and Sharma CB: Bilirubin peak can be mistaken as $\mathrm{Hb}$ Bart's or $\mathrm{Hb} \mathrm{H}$ on High-performance liquid chromatography. Hemoglobin 35: 171-174, 2011.

18. Tao YF, Deng ZF, Liao L, Qiu YL, Chen WQ and Lin FQ: Comparison and evaluation of three screening tests of hereditary spherocytosis in Chinese patients. Ann Hematol 94: 747-751, 2015.

19. Lazarova E, Pradier O, Cotton F and Gulbis B: Automated reticulocyte parameters for hereditary spherocytosis screening. Ann Hematol 93: 1809-1818, 2014.

20. Xu Y, Yang W, Liao L, Deng Z, Qiu Y, Chen W and Lin F: Mean reticulocyte volume: A specific parameter to screen for hereditary spherocytosis. Eur J Haematol 96: 170-174, 2016.

21. Deng Z, Liao L, Yang W and Lin F: Misdiagnosis of two cases of hereditary spherocytosis in a family and review of published reports. Clin Chim Acta 441: 6-9, 2015.

22. King MJ and Zanella A: Hereditary red cell membrane disorders and laboratory diagnostic testing. Int J Lab Hematol 35: 237-243, 2013.

23. Wang J, Zheng B, Zhao Y, Chen X, Liu Y, Bo L, Zheng Y, Zhang $\mathrm{F}, \mathrm{Ru} \mathrm{K}$ and Wang $\mathrm{H}$ : Flow cytometric test using eosin5'-maleimide (EMA) labelling of red blood for diagnosis of hereditary spherocytosis. Zhonghua Xue Ye Xue Za Zhi 36: 598-601, 2015 (In Chinese).

24. Suemori S, Wada H, Nakanishi H, Tsujioka T, Sugihara T and Tohyama K: Analysis of hereditary elliptocytosis with decreased binding of eosin-5-maleimide to red blood cells. BioMed Res Int 2015: 451861, 2015.
25. Joshi P, Aggarwal A, Jamwal M, Sachdeva MU, Bansal D, Malhotra P, Sharma P and Das R: A comparative evaluation of Eosin-5'-maleimide flow cytometry reveals a high diagnostic efficacy for hereditary spherocytosis. Int J Lab Hematol 38: 520-526, 2016.

26. Perrotta S, Della Ragione F, Rossi F, Avvisati RA, Di Pinto D, De Mieri G, Scianguetta S, Mancusi S, De Falco L, Marano V, et al: Beta-spectrinBari: A truncated beta-chain responsible for dominant hereditary spherocytosis. Haematologica 94: 1753-1757, 2009.

27. Serpillon S, Floyd BC, Gupte RS, George S, Kozicky M, Neito V, Recchia F, Stanley W, Wolin MS and Gupte SA: Superoxide production by NAD(P)H oxidase and mitochondria is increased in genetically obese and hyperglycemic rat heart and aorta before the development of cardiac dysfunction. The role of glucose-6phosphate dehydrogenase-derived NADPH. Am J Physiol Heart Circ Physiol 297: H153-H162, 2009.

28. Perrotta S, Gallagher PG and Mohandas N: Hereditary spherocytosis. Lancet 372: 1411-1426, 2008.

29. Da Costa L, Galimand J, Fenneteau O and Mohandas N: Hereditary spherocytosis, elliptocytosis, and other red cell membrane disorders. Blood Rev 27: 167-178, 2013.

30. Adzhubei I, Jordan DM and Sunyaev SR: Predicting functional effect of human missense mutations using PolyPhen-2. Curr Protoc Hum Genet Chapter 7: Unit7.20, 2013.

31. Maréchal J, Wilmotte R, Kanzaki A, Dhermy D, Garbarz M, Galand C, Tang TK, Yawata Y and Delaunay J: Ethnic distribution of allele alpha LELY, a low-expression allele of red-cell spectrin alpha-gene. Br J Haematol 90: 553-556, 1995

32. Randon J, Boulanger L, Marechal J, Garbarz M, Vallier A, Ribeiro L, Tamagnini G, Dhermy D and Delaunay J: A variant of spectrin low-expression allele alpha LELY carrying a hereditary elliptocytosis mutation in codon 28. Br J Haematol 88: 534-540, 1994.

33. Tolpinrud W, Maksimova YD, Forget BG and Gallagher PG: Nonsense mutations of the alpha-spectrin gene in hereditary pyropoikilocytosis. Haematologica 93: 1752-1754, 2008. 